\title{
Orbital elements and physical parameters of ten chromospherically active binary stars $^{\star}$
}

\author{
F.C. Fekel ${ }^{1, \star \star}$, K.G. Strassmeier ${ }^{2, \star \star, \star \star \star, \dagger}$, M. Weber ${ }^{2, \star \star, \star \star \star}$, and A. Washuettl ${ }^{2, \star \star, \star \star \star}$ \\ 1 Center for Automated Space Science and Center of Excellence in Information Systems, Tennessee State University, \\ Nashville, TN 37203, U.S.A. \\ e-mail: fekel@coe.tnstate.edu \\ ${ }^{2}$ Institut für Astronomie, Universität Wien, Türkenschanzstraße 17, A-1180 Wien, Austria \\ e-mail: strassmeier@astro.univie.ac.at, weber@astro.univie.ac.at, wasi@astro.univie.ac.at
}

Received March 17; accepted April 15, 1999

\begin{abstract}
Orbital elements have been determined for 10 chromospherically active binaries from a combination of new velocities and velocities in the literature. First orbits for three binaries, HD 33363, HD 152178, and HD 208472, are presented, as well as updated orbits for seven other binaries. Two of the latter systems, LN Peg and HD 106225 were discovered to be triple, and both short- and longperiod orbits have been computed for each. Fundamental properties have been determined for the chromopherically active primary in each system.
\end{abstract}

Key words: stars: binaries: spectroscopic; binaries: general — stars: late-type — stars: fundamental parameters — stars: activity

\section{Introduction}

The interplay of convection and rapid rotation results in chromospheric activity in late-type stars. For such stars rapid rotation occurs as the result of youth, before a stellar wind slows its rotation, or duplicity, where tidal forces

\footnotetext{
* Appendix A and Tables A1 to A15 only available in electronic form at the CDS via anonymous $\mathrm{ftp}$ to cdsarc.u-strasbg.fr (130.79.128.5) or via http://cdsweb.ustrasbg.fr/Abstract.html

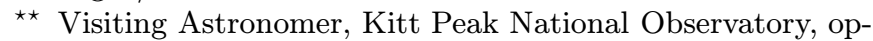
erated by the Association of Universities for Research in Astronomy, Inc. under contract with the National Science Foundation.

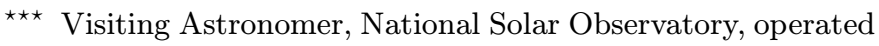
by the Association of Universities for Research in Astronomy, Inc. under contract with the National Science Foundation.

$\dagger$ Visiting astronomer, European Southern Observatory, LaSilla, Chile.
}

cause the rotation of the stars to become synchronized. The "Catalog of Chromospherically Active Binary Stars (second edition)" (CABS catalog; Strassmeier et al. 1993) lists basic information, including orbital elements and fundamental parameters, for over 200 active binaries.

Evidence of the dynamo, which is believed to be generated by the interaction of magnetic fields and rotation, includes Ca II $\mathrm{H}$ and $\mathrm{K}$ emission lines and starspots. Some chromospherically active binaries are rotating so rapidly that the distribution of starspots can be mapped on their surfaces with the Doppler imaging technique (Vogt \& Penrod 1983). In the course of such a project being carried out by the research group at the University of Vienna (e.g. Strassmeier \& Rice 1998), a number of chromospherically active binaries have been observed spectroscopically for several seasons. To produce an accurate spot map the data must be correctly phased, so orbital elements should be carefully determined or updated. Thanks to back-toback observing runs, arranged at the Kitt Peak National Observatory (KPNO) by the telescope-allocation committee, it was learned that additional spectra of many of the stars have been obtained by one of us (FCF), and so it was decided to combine our data.

In this paper we determine initial orbital elements for three binaries and update the elements of seven others. In the process we have found evidence of a tertiary component in two of the systems. The availability of the Hipparcos-parallax data base has enabled us to determine improved fundamental properties for the 10 systems.

\section{New observations}

Our spectroscopic observations of the 10 program stars were obtained at several observatories including the McDonald Observatory of the University of Texas, the KPNO, and the National Solar Observatory (NSO), all 
Table 1. Program stars

\begin{tabular}{|c|c|c|c|c|c|c|c|c|c|c|}
\hline Star & $\begin{array}{l}\text { Variable } \\
\text { name }\end{array}$ & $\begin{array}{l}V^{a} \\
\text { (mag) }\end{array}$ & $\begin{array}{l}B-V^{a} \\
(\mathrm{mag})\end{array}$ & $\begin{array}{l}V-I^{a} \\
(\mathrm{mag})\end{array}$ & $\begin{array}{l}\text { Spectral } \\
\text { type }\end{array}$ & SB & $\begin{array}{l}P_{\text {orb }} \\
\text { (days) }\end{array}$ & $\begin{array}{l}<P_{\text {rot }}> \\
\text { (days) }\end{array}$ & $\begin{array}{l}v \sin i^{b} \\
\left(\mathrm{~km} \mathrm{~s}^{-1}\right)\end{array}$ & Variable type \\
\hline $\mathrm{BD}+13^{\circ} 13$ & LN Peg & 8.44 & 0.739 & 0.79 & G8 V & SB2 & 1.84 & 1.84 & 23.7 & RS CVn \\
\hline HD 4502 & $\zeta$ And & 4.08 & 1.100 & 1.06 & K1 IIIa & SB1 & 17.7 & 17.5 & 41 & RS CVn + ell. \\
\hline HD 33363 & CL Cam & 7.42 & 1.072 & 1.04 & K0 III & SB1 & 20.8 & 41.5 & 10.7 & RS CVn \\
\hline HD 81410 & IL Hya & 7.35 & 1.012 & 0.99 & K1 III & SB2 & 12.9 & 12.78 & 26.5 & RS CVn \\
\hline HD 106225 & HU Vir & 8.70 & 0.974 & 0.96 & K0 III-IV & SB1 & 10.3 & 10.42 & 25 & RS CVn \\
\hline HD 136901 & UV CrB & 7.23 & 1.244 & 1.20 & K2 III & SB1 & 18.6 & 18.8 & 42 & RS CVn + ell. \\
\hline $\mathrm{BD}-08^{\circ} 3999$ & UZ Lib & 9.10 & 1.025 & 1.00 & K0 III & SB1 & 4.76 & 4.75 & 67 & RS CVn \\
\hline HD 152178 & V2253 Oph & 8.55 & 1.095 & 1.06 & K0 III & SB1 & 314 & 22.35 & 28.8 & RS CVn \\
\hline HD 208472 & V2075 Cyg & 7.49 & 1.066 & 1.03 & G8 III & SB1 & 22.6 & 22.54 & 19.7 & RS CVn \\
\hline HD 216489 & IM Peg & 5.86 & 1.132 & 1.10 & K2 II-III & SB1 & 24.6 & 24.49 & 28.2 & RS CVn \\
\hline
\end{tabular}

${ }^{a}$ From Hipparcos (ESA 1997).

${ }^{b}$ Errors are typically $1 \mathrm{~km} \mathrm{~s}^{-1}$.

in the northern hemisphere, as well as the European Southern Observatory (ESO) in the southern hemisphere. Table 1 lists basic data for our program stars, and Fig. 1 shows a sample spectrum of each star.

Table 2 summarizes the telescope/detector configurations used. However, since the vast majority of the spectrograms were obtained between 1983 and 1998 at the KPNO with the coudé feed telescope, coudé spectrograph, a couple combinations of grating and camera, and several different CCD detectors, primarily a Texas Instruments (TI) or Ford (F3KB) CCD detector, those particular configurations are discussed in more detail below.

From 1983 through 1987 the $800 \times 800$ TI CCD with $15 \mu$ pixels was used with grating $\mathrm{D}$ and camera 5 , to yield a resolving power, $R=30000$. The spectra have a $90 \AA$ wavelength range centered on $6430 \AA$ with an effective wavelength resolution of $0.23 \AA$. Improved throughput was obtained when KPNO acquired grating A, which has been used from 1987 through 1998 with camera 5, the long collimator, and a TI CCD to obtained a slightly better resolution of $0.21 \AA$, while reducing the wavelength range to $82 \AA$. From 1995 through 1998 additional observations were obtained with the $3096 \times 1024$ F3KB CCD $(15 \mu$ pixels) but an otherwise identical spectrograph setup. For this combination the wavelength range is $300 \AA$, the effective wavelength resolution is $0.27 \AA$, and the spectra were centered at $6480 \AA$.

Data from the coudé feed have recently been supplemented with observations obtained at two other observatories. The NSO observations were obtained with the McMath-Pierce solar telescope during a single 70-night observing run from November 1, 1996 through January 8, 1997. Another TI CCD was used in conjunction with Milton-Roy grating \# 1 to give an effective wavelength resolution of $0.15 \AA$ and a usable wavelength range of $55 \AA$ centered at $6435 \AA$.

For two stars, UZ Librae and HD 152178, observations also were obtained at ESO La Silla during seven
Table 2. Telescope-spectrograph-detector combinations

\begin{tabular}{|c|c|c|c|c|c|}
\hline Telescope & Years & Detector & $\begin{array}{c}\text { Resol. } \\
(\AA)\end{array}$ & $\begin{array}{l}\Delta \lambda \\
(\AA)\end{array}$ & $\begin{array}{l}\text { Source } \\
\text { code }\end{array}$ \\
\hline $\begin{array}{l}\text { McDonald } \\
2.7 \mathrm{~m}\end{array}$ & $1979-81$ & Reticon & 0.36 & 110 & $\mathrm{MR}$ \\
\hline $\begin{array}{l}\text { KPNO } \\
\text { coudé feed }\end{array}$ & 1982 & $\begin{array}{l}\text { Fairchild } \\
\text { CCD }\end{array}$ & 0.45 & 165 & KFA \\
\hline $\begin{array}{l}\text { KPNO } \\
\text { coudé feed }\end{array}$ & $1982-83$ & $\begin{array}{l}\text { RCA } \\
\text { CCD }\end{array}$ & 0.30 & 65 & $\mathrm{KR}$ \\
\hline $\begin{array}{l}\text { KPNO } \\
\text { coudé feed }\end{array}$ & $1983-87$ & $\begin{array}{l}\text { TI } \\
\text { CCD }\end{array}$ & 0.23 & 90 & KT1 \\
\hline $\begin{array}{l}\text { KPNO } \\
\text { coudé feed }\end{array}$ & 1987-98 & $\begin{array}{l}\text { TI } \\
\text { CCD }\end{array}$ & 0.21 & 82 & KT2 \\
\hline $\begin{array}{l}\text { KPNO } \\
\text { coudé feed }\end{array}$ & 1995-98 & $\begin{array}{l}\text { Ford } \\
\text { CCD }\end{array}$ & 0.27 & 300 & $\mathrm{KF}$ \\
\hline $\begin{array}{l}\text { NSO } \\
\text { McMath-P. }\end{array}$ & 1996-97 & $\begin{array}{l}\text { TI } \\
\text { CCD }\end{array}$ & 0.15 & 55 & NSO \\
\hline $\begin{array}{l}\mathrm{ESO} \\
1.4 \mathrm{~m} \mathrm{CAT}\end{array}$ & 1996 & $\begin{array}{l}\text { Loral } \\
\text { CCD }\end{array}$ & 0.09 & 70 & ESO \\
\hline
\end{tabular}

nights, from May 18-23, 1996. The 1.4 m coudé auxiliary telescope (CAT) was used with the coudé echelle spectrograph (CES) operating in single-order mode. This spectrograph setup together with a $2688 \times 512$ Loral CCD having $15 \mu$ pixels resulted in $R=70000$. The spectra have a wavelength range of about $70 \AA$ and are centered at $6425 \AA$.

Bias subtraction, flat-field division, wavelength calibration, and continuum rectification were performed on the raw spectra with the programs in IRAF (distributed by NOAO) or MIDAS (distributed by ESO). Thoriumargon comparison spectra were obtained each night at intervals of one to two hours to ensure an accurate wavelength calibration. 


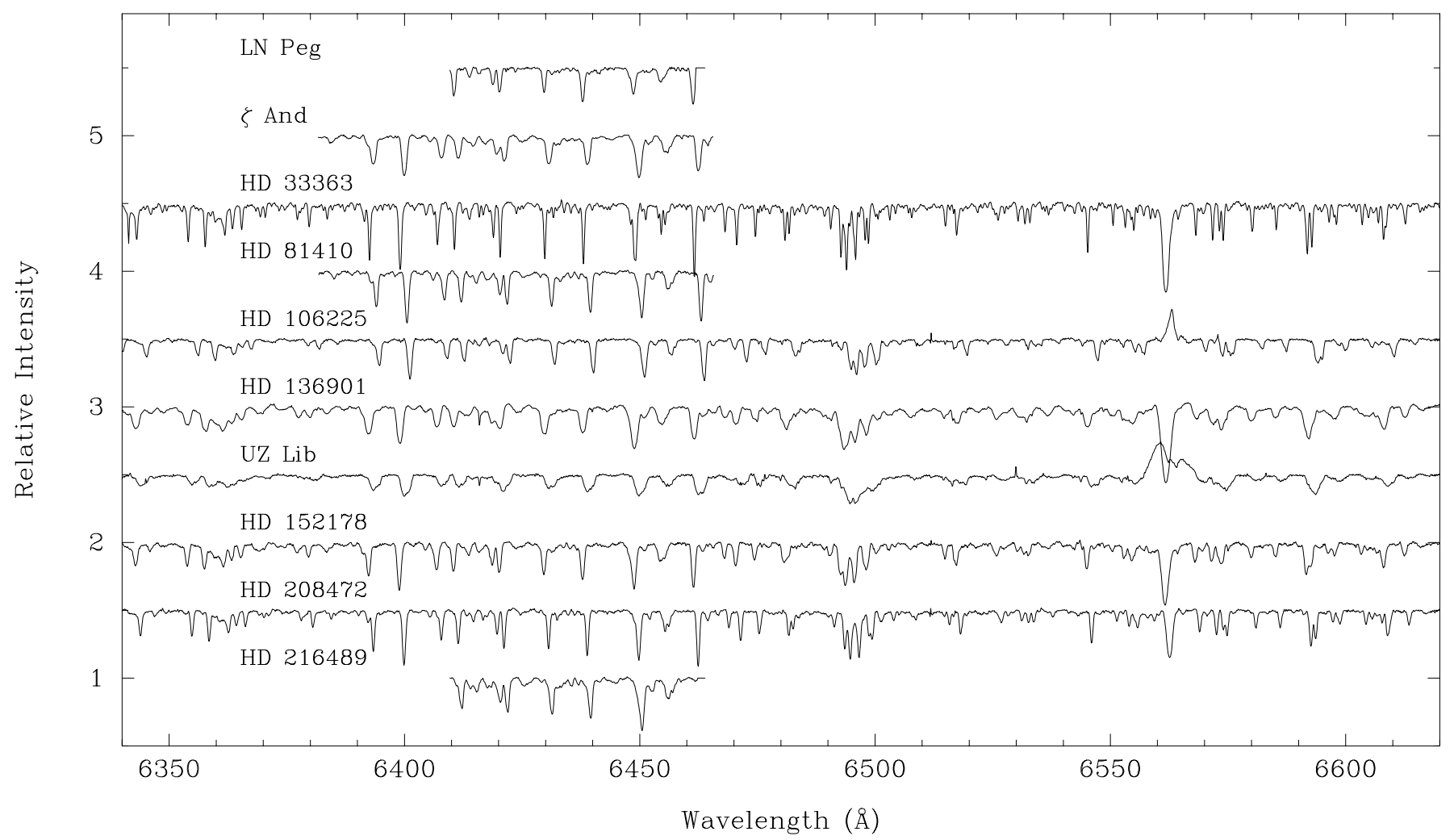

Fig. 1. Examples of spectra used in the analysis of this paper. The different wavelength coverages are due to different telescope/detector combinations and range from $55 \AA$ for, e.g., LN Peg at NSO to $300 \AA$ for most other stars

\section{Velocity reductions and orbit computations}

Radial velocities of the program stars were determined with the IRAF cross-correlation program fxcor (see Fitzpatrick 1993). Several different IAU radial-velocity standard stars were observed during the course of each night at intervals of one to two hours. Their radial velocities were assumed from the work of Scarfe et al. (1990). Other stars used as standards included $\mu$ Her A, assumed velocity of $-16.4 \mathrm{~km} \mathrm{~s}^{-1}$ (Stockton \& Fekel 1992) and $\beta$ Aql, assumed velocity of $-40.2 \mathrm{~km} \mathrm{~s}^{-1}$, from extensive unpublished coudé feed results. The velocities of both stars are tied to the Scarfe et al. (1990) system. The small number of ESO velocities were measured relative to $\beta \mathrm{Crv}=\mathrm{HR} 4786$, assumed velocity of $-7.6 \mathrm{~km} \mathrm{~s}^{-1}$ (Udry 1998).

Although the velocities of our standards are on a welldefined system, the combination of our various new data sets into a single radial-velocity solution is complicated because of the spectral-type mismatch of the standard and program stars and the different and relatively small wavelength ranges covered by the spectra (from $55 \AA$ to $300 \AA$ ), as well as the strong chromospheric activity and large $v \sin i$ of many of the program stars. Unless the spectral type of a standard star is very similar to that of the program star, line strengths, particularly of the weak and moderate strength features, may differ significantly in the program and standard star. Since our program stars are rotationally broadened, such differences in line strength will cause different amounts of blending with nearby strong lines. Different spectrograph setups, resulting in different observed wavelength ranges, exacerbate the line-strength blending problem associated with any spectral-type mismatch. Despite such potential problems, we found that the zero-point velocity shifts between our data sets were not significant, except for the most chromospherically active stars, whose line profiles show obvious variability due to starspots rotating in and out of view.

When the number of observations warranted it, independent orbital solutions were obtained of our various data sets, as well as solutions of data sets in the literature. Often the errors of the older velocities are quite large compared to those of our velocities. In such cases the older velocities were of little use except to improve the period and so were not usually included in the final orbital solution. Furthermore, if the $\mathrm{O}-\mathrm{C}$ residual of one of our observation exceeded 3.0 sigma it was given zero weight in the final solution but is still listed in the tables in the Appendix.

Several different computer programs, which were used to determine the orbital elements of the various systems, are mentioned in the individual orbital-elements sections. Preliminary elements often were determined with BISP, a computer program that uses a slightly modified 
version of the Wilsing-Russell method (Wolfe et al. 1967). A differential-corrections program, called SB1, of Barker et al. (1967) has been used to compute eccentric orbits of single-lined systems. For some systems the orbital eccentricity is small enough that a circular-orbit solution may be appropriate. Circular orbits for single- and doublelined binaries have been computed with SB1C and SB2C (Barlow 1998), respectively, both of which use differential corrections to determine the elements. For triple systems a general least-squares (GLS) program (Daniels 1966) has been used to solve simultaneously for the elements of the short- and long-period orbits.

\section{Determination of fundamental parameters}

The advent of new or vastly improved parallaxes from the Hipparcos satellite (ESA 1997) has enabled us to calculate significantly improved fundamental parameters for our 10 systems. Such values clearly supersede those given in Table 5 of the CABS catalog (Strassmeier et al. 1993). The literature was searched for the brightest known magnitude of each system, which was assumed to be the unspotted $V$ magnitude of each primary. For the two double-lined systems, LN Peg and HD 81410 (Fig. 2), we converted the equivalent-width ratio of the primary to secondary spectral lines into a brightness difference and corrected the combined magnitude. For LN Peg the secondary's contribution is approximately 0.25 in $V$ and for HD 81410, $0 . \mathrm{m} 1$. Then an $M_{V}$ was computed from the Hipparcos parallax. For the eight systems closer than 160 pc no interstellar reddening was assumed. However, because of their much greater distances, a reddening of 0.2 was assumed for HD 136901 and 0.4 was assumed for HD 152178. Except for HD 136901 and HD 152178, whose $B-V$ values were revised with $R=3.2$ and the above reddening, the Hipparcos $B-V$ values given in Table 1 were used in conjunction with Table 3 of Flower (1996) to obtain a bolometric correction, B.C., and effective temperature, $T_{\text {eff }}$, for each primary. An $M_{\text {bol }}$ was computed for each star and converted into units of solar luminosity assuming $M_{\mathrm{bol}}=+4 .{ }^{\mathrm{m}} 64$ for the Sun (Schmidt-Kaler 1982). Finally, a radius, called $R_{\text {Hip }}$, was determined for each star from the $T_{\text {eff }}$ and $L / L \odot$ values. It was assumed that the errors in the computed quantities are dominated by errors in the parallax, given in the Hipparcos catalog, and to a lesser extent by the $T_{\text {eff }}$, with the latter error estimated to be $\pm 100 \mathrm{~K}$.

For each active primary a value of $R \sin i$, which is independent of the $R_{\text {Hip }}$ value, was computed from its rotational period and $v \sin i$ value listed in Table 1. For $R \sin i$ the error is dominated by the uncertainty in $v \sin i$. Table 3 summarizes the fundamental parameters for our stars.

The values of $R \sin i$, of course, should be smaller than the corresponding values of $R_{\text {Hip }}$, but this is not the case for the computed values of the majority of our stars (Table 3). Within the errors, however, agreement between $R \sin i$ and $R_{\text {Hip }}$ is acceptable for eight of the 10 stars. For two stars, UZ Lib and HD 106225, the $R_{\text {Hip }}$ values are significantly smaller than those computed for $R \sin i$. Their Hipparcos distances result in $M_{V}$ and $R_{\text {Hip }}$ values that indicate that the stars are subgiants, while the minimum radii suggest that both stars have at least begun to ascend the red-giant branch. Note that if we increase the error in $T_{\text {eff }}$ from $\pm 100 \mathrm{~K}$ to $\pm 200 \mathrm{~K}$, the error in $R_{\text {Hip }}$ increases by $15-20 \%$ but is still only $30 \%$ the error from the parallax.

For UZ Lib the Hipparcos distance is nearly a factor of four smaller than the value used by Grewing et al. (1989), which led to contradictory results described by Strassmeier (1996) as the dilemma of big leg Emma (Zappa 1974). However, as a result of the improved distance, we have not extricated ourselves from the dilemma. Instead, it has reversed itself with the radius now being too small compared to the minimum radius.

Systematic trends between $R \sin i$ and $R_{\text {Hip }}$, and in particular the problems with the values for UZ Lib and HD 106225, are likely the result of several different factors. One obvious contributor is our assumption that the brightest known magnitude of each system is equal to its unspotted magnitude. For example, for the very active star II Peg, O'Neal et al. (1996) compared photometric and spectroscopic results and found "a substantial symmetric spotted component," which they attributed to either a polar spot or spots evenly distributed in longitude. Thus, our assumed unspotted magnitudes listed in Table 3 are almost certainly too faint. For II Peg, O'Neal et al. (1996) computed $V=6 \cdot 8$ compared with the historically observed $V_{\max }=7 \cdot 2$ (Strassmeier et al. 1993). In the cases of UZ Lib and HD 106225, if the unspotted magnitude were $0 .{ }^{\mathrm{m}} 5$ brighter than our assumed value, the $R_{\text {Hip }}$ values would be increased by $0.9 R_{\odot}$.

\section{5. $\mathrm{BD}+13^{\circ} 13=$ SAO $91772=\mathrm{LN}$ Peg}

\subsection{Brief history}

LN Peg was identified as a chromospherically active binary by Bergoffen et al. (1988), who discovered the strong Ca II K emission and radial-velocity variations of this star. Latham et al. (1988) computed a single-lined circular orbit with a period of 1.844 days. However, Pasquini \& Lindgren (1994) detected Ca II K emission from both the primary and the secondary.

Rodonó et al. (1994) discovered the system's light variability and from their few observations found a period consistent with the orbital period. From photometry obtained during two observing seasons, Henry et al. (1995) identified four possible periods. They concluded that the one at 1.852 days represents the rotational period because it most closely agrees with the orbital period. 
Table 3. Fundamental parameters

\begin{tabular}{lllllllll}
\hline Star & $\begin{array}{l}V_{\text {unspotted }} \\
(\mathrm{mag})\end{array}$ & $\begin{array}{l}T_{\text {eff }}^{a} \\
(\mathrm{~K})\end{array}$ & $\begin{array}{l}\text { B.C. }^{a} \\
(\mathrm{mag})\end{array}$ & $\begin{array}{l}\text { Dist. } \\
(\mathrm{pc})\end{array}$ & $\begin{array}{l}M_{V} \\
(\mathrm{mag})\end{array}$ & $\begin{array}{l}L \\
\left(L_{\odot}\right)\end{array}$ & $\begin{array}{l}R_{\text {Hip }} \\
\left(R_{\odot}\right)\end{array}$ & $\begin{array}{l}R \sin i \\
\left(R_{\odot}\right)\end{array}$ \\
\hline LN Peg Aa & 8.65 & 5444 & -0.153 & 40.5 & +5.64 & 0.46 & 0.76 & 0.86 \\
& & \pm 100 & & \pm 1.9 & \pm 0.10 & \pm 0.04 & \pm 0.06 & \pm 0.04 \\
S And & 4.05 & 4658 & -0.494 & 55.6 & +0.32 & 84 & 14.1 & 14.2 \\
& & \pm 100 & & \pm 2.4 & \pm 0.09 & \pm 7 & \pm 1.2 & \pm 0.4 \\
HD 33363 & 7.34 & 4711 & -0.461 & 137 & +1.66 & 23.9 & 7.4 & 8.8 \\
& & \pm 100 & & \pm 12 & \pm 0.19 & \pm 4.2 & \pm 1.0 & \pm 0.8 \\
HD 81410 A & 7.35 & 4823 & -0.394 & 120 & +1.95 & 17.1 & 5.9 & 6.7 \\
& & \pm 100 & & \pm 11 & \pm 0.20 & \pm 3.1 & \pm 0.8 & \pm 0.3 \\
HD 106225 Aa & 8.57 & 4896 & -0.356 & 125 & +3.08 & 5.8 & 3.4 & 5.1 \\
& & \pm 100 & & \pm 17 & \pm 0.30 & \pm 1.6 & \pm 0.6 & \pm 0.2 \\
HD 136901 & 7.19 & 4516 & -0.589 & 279 & -0.24 & 154 & 20.3 & 15.6 \\
& & \pm 100 & & \pm 55 & \pm 0.43 & \pm 63 & \pm 5.0 & \pm 1.2 \\
UZ Lib & 8.90 & 4801 & -0.408 & 140 & +3.17 & 5.6 & 3.4 & 6.28 \\
& & \pm 100 & & \pm 22 & \pm 0.34 & \pm 1.8 & \pm 0.7 & \pm 0.07 \\
HD 152178 & 8.43 & 4902 & -0.352 & 472 & -0.34 & 136 & 16.2 & 12.7 \\
& & \pm 100 & & \pm 174 & \pm 0.84 & \pm 116 & \pm 7.1 & \pm 0.5 \\
HD 208472 & \multirow{2}{*}{7.36} & 4719 & -0.453 & 157 & +1.38 & 30.5 & 8.3 & 8.8 \\
& & \pm 100 & & \pm 16 & \pm 0.22 & \pm 6.3 & \pm 1.2 & \pm 0.4 \\
HD 216489 & 5.64 & 4600 & -0.529 & 96.8 & +0.71 & 61 & 12.3 & 13.6 \\
& & \pm 100 & & \pm 6.6 & \pm 0.15 & \pm 8 & \pm 1.4 & \pm 0.5 \\
\hline
\end{tabular}

${ }^{a}$ From $B-V$ according to Flower (1996).

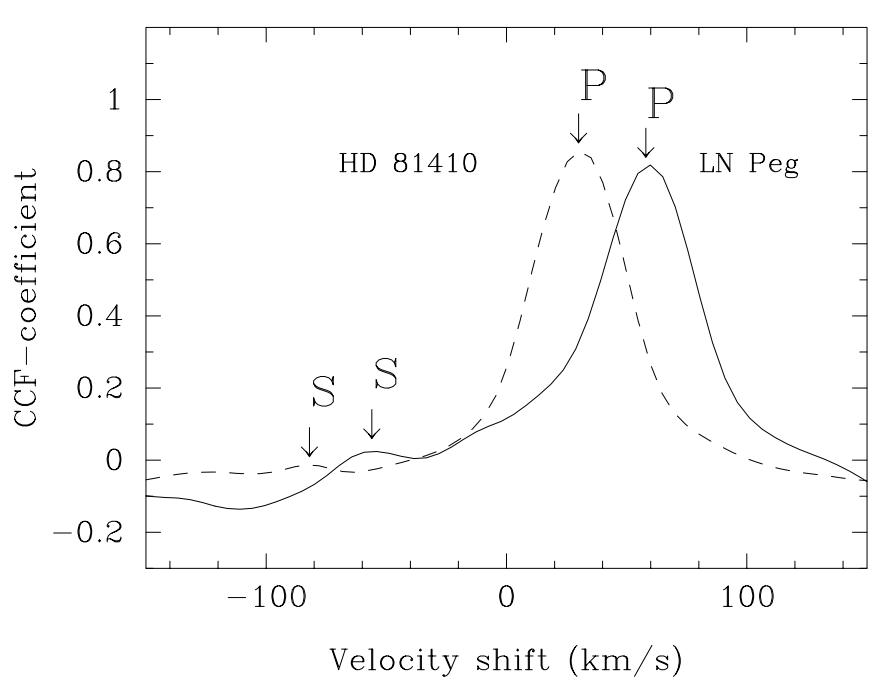

Fig. 2. The cross-correlation functions (CCFs) for the two double-lined systems, LN Peg (solid line) and HD 81410 (dashed line). Arrows indicate the primary (P) and the secondary (S) CCF for each system

\subsection{Orbital elements}

The primary and secondary components of LN Peg seen in our spectra are called $\mathrm{Aa}$ and $\mathrm{Ab}$, respectively, while the two stars together are called component A.

Two new sets of observations have been obtained for LN Peg. The Vienna group collected 39 spectra during a single, extensive 1996-1997 observing run at the NSO.
From November 1993 through October 1998, FCF obtained 13 observations at KPNO. Also available for analysis are the velocities of Latham et al. (1988) and three velocities of Fleming et al. (1989), which are on the same velocity system.

Assuming the orbital period of Latham et al. (1988), a preliminary set of orbital elements was determined for the NSO velocities of the primary, component Aa, with BISP and refined with SB1. Agreement with the orbital elements of Latham et al. (1988) was excellent except for the center-of-mass velocity, which was $7 \mathrm{~km} \mathrm{~s}^{-1}$ more negative, suggesting that the system is triple. This possibility was confirmed when a combined solution of the NSO and KPNO data produced systematic velocity residuals.

An SB1 solution of all the velocities of Aa, but with zero weight given to the Latham et al. (1988), Fleming et al. (1989), and KPNO data, resulted in residuals that were analyzed to determine the period of the long-term velocity variations. Because of the spacing of the data, two possible periods emerged, 4.3 and 8.6 years. With BISP a set of preliminary long-period orbital elements was computed for each period. Then, with one velocity given zero weight, GLS was used to obtain a simultaneous solution of the short- and long-period orbits. Within their errors, the orbital elements of the short-period orbit are the same for both solutions. However, the long period orbits are rather different. For example, the 4.3-year period has $e=0.054 \pm 0.064$ while the 8.6-year period has $e=0.49 \pm 0.05$. Since the weighted sum of the residuals 

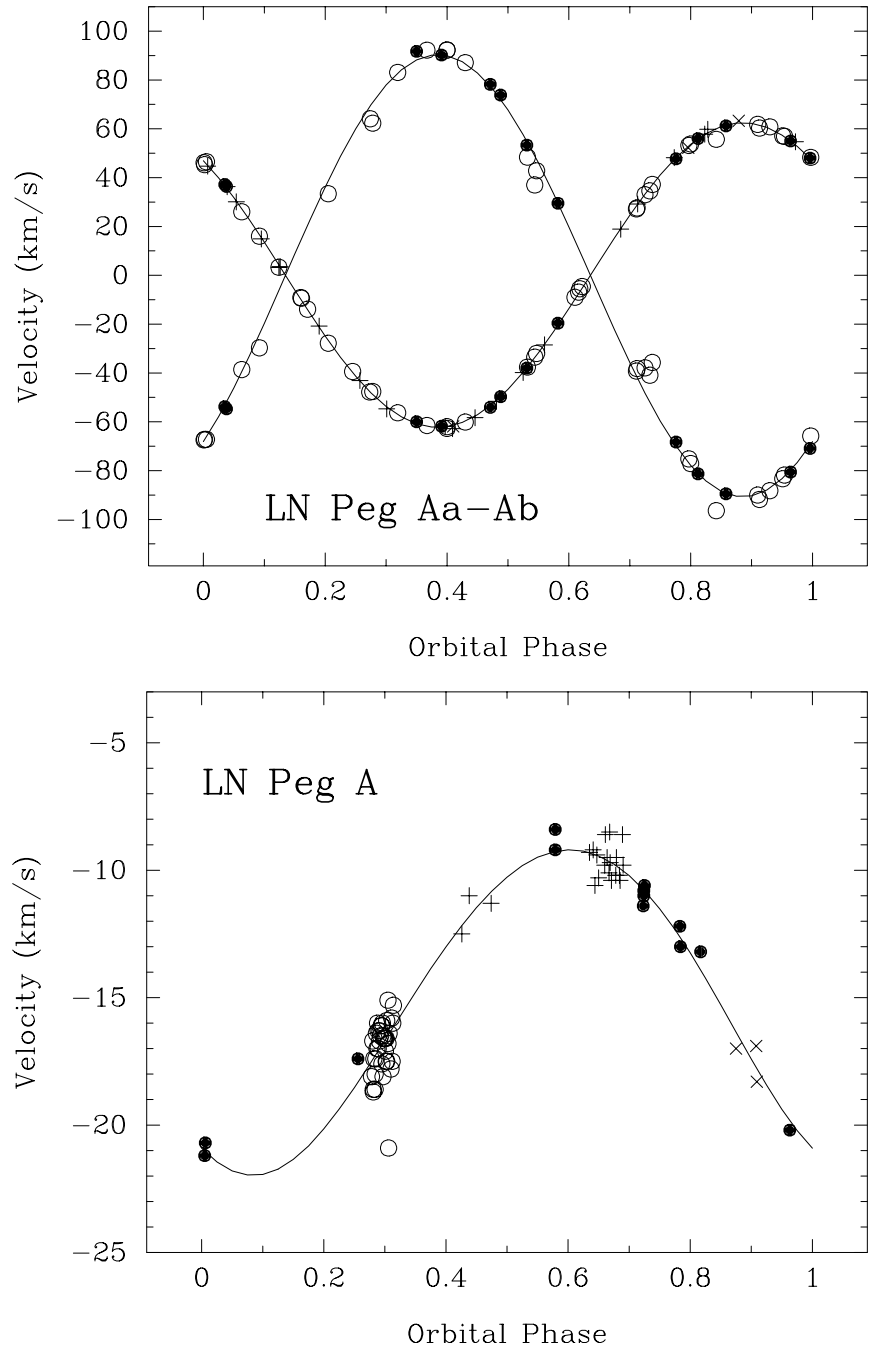

Fig. 3. Radial-velocity curves of LN Peg. The upper panel is for the short-period orbit $(\mathrm{Aa}-\mathrm{Ab})$, the lower panel for the long-period orbit of LN Peg A (only for Aa). Open symbols are NSO McMath-Pierce data, filled symbols are from KPNO, pluses from Latham et al. (1988), and crosses from Fleming et al. (1989)

squared for the 8.6-year solution is $15 \%$ larger than for the 4.3-year solution, we have assumed that the 4.3-year solution is correct. However, we note that orbits with such long periods have no propensity toward zero eccentricity, so additional observations will be necessary for a definitive decision.

Absorption lines of the secondary, component Ab, are visible at red wavelengths. They are also rotationally broadened but are much weaker than those of Aa, making velocity measurements difficult. Thus, a GLS solution of the NSO and KPNO velocities of $\mathrm{Ab}$ was obtained with only the short-period semiamplitude varied. All other orbital elements were fixed at the values obtained for Aa. Six NSO velocities with residuals greater than $9 \mathrm{kms}^{-1}$ were given zero weight.

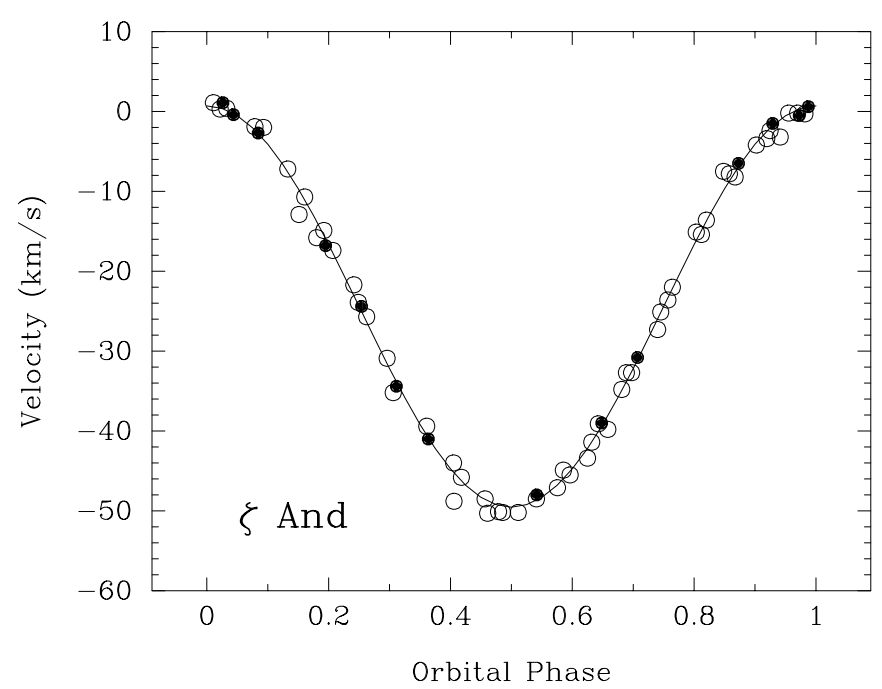

Fig. 4. Radial-velocity curve of $\zeta$ And. Open symbols are NSO McMath-Pierce data, filled symbols are from KPNO. Zero phase is the time of maximum positive velocity

The short- and long-period orbital elements are given in Table 5 . The short-period eccentricity is quite small $0.054 \pm 0.064$, but has been retained because in triple systems the third star may cause a non-zero eccentricity (Mazeh \& Shaham 1979; Söderhjelm 1984) in the short-period orbit. For the tables and figures each measured velocity has been separated into a short- and long-period component, which consists of the shortor long-period calculated velocity plus one-half of the computed residual. Thus, the sum of those separated short- and long-period velocities for each date of observation results in the actually measured velocity. The short-period velocities and residuals to the computed fit are listed for both components in Tables A1 and A2 in the (electronic) Appendix, respectively, while those for the long period are in Tables A3 and A4, respectively. Included in those tables are the velocities of Latham et al. (1988) and Fleming et al. (1989). The computed short-period orbit compared with our velocities is shown in the upper panel of Fig. 3, while a similar comparison for the long-period velocities of the primary is in the lower panel of Fig. 3. For the short-period orbit a time of conjunction with the primary behind the secondary is HJD 2446348.080 . The standard error of an observation of unit weight is $1.0 \mathrm{~km} \mathrm{~s}^{-1}$.

Our results for LN Peg demonstrate that new observations are valuable not only to confirm old orbital elements but also to detect additional components. This is particularly true when an initial orbital solution has been determined from observations obtained over only one or two observing seasons. 


\section{6. $\zeta$ And $=$ HR $215=$ HD 4502}

\subsection{Brief history}

Campbell (1911) was the first to report the variable velocity of $\zeta$ And and also commented on the obvious broadening of its absorption lines. Shortly thereafter, Cannon (1915) computed the initial set of orbital elements for this bright star. Spencer Jones (1928) and Gratton (1950) obtained additional velocities, and each determined a new orbital solution. The latter's remained the standard for nearly 50 years. Recently however, Kaye et al. (1995) recomputed a new orbital solution from 135 velocities from the literature.

The Ca II emission lines of $\zeta$ And were independently discovered by Joy \& Wilson (1949) and Gratton (1950). The latter also noted that the absorption lines are diffuse, suggesting a noticeable rotational velocity. From new spectrograms Hendry (1980) discussed the variability of the emission lines.

Light variations, initially discovered by Stebbins (1928), are primarily the result of the ellipticity effect (e.g. Stebbins 1928; Strassmeier et al. 1989). Variability due to starspots with a period similar to the orbital period and an amplitude as great as 0.04 has also been detected (Kaye et al. 1995). We note that the maximum spot amplitude of 0 . 083 , highlighted by Kaye et al. (1995), appears to be a misprint.

\subsection{Orbital elements}

For $\zeta$ And two new sets of velocities have been obtained, 53 velocities at NSO during an extensive 1996-1997 observing run, and 14 velocities at KPNO during a single run in 1997-1998. With the orbital period fixed at Gratton's (1950) value and his other elements used as starting values, independent orbital solutions of the two data sets were carried out with the SB1 computer program. The results indicated no need for a zero-point shift, and the two sets were combined with weights of 1.0 for the KPNO velocities and 0.5 for the NSO velocities. Independent solutions were also computed for the large data sets of Cannon (1915) and Spencer Jones (1928), resulting in weights of 0.02 and 0.03 , respectively, and a velocity offset of $6 \mathrm{~km} \mathrm{~s}^{-1}$, which was added to the data of Cannon (1915). Additional sets of velocities in the literature, including those of Gratton (1950), do not warrant inclusion. They generally contain just a few velocities and have very low weights, providing no additional leverage on the period or other elements.

An SB1 orbital solution with the four sets of appropriately weighted velocities resulted in an eccentricity of $0.0068 \pm 0.0056$. Because of this very low eccentricity, a circular-orbit solution for the same data was computed with SB1C. According to the precepts of Lucy \& Sweeney (1971), the circular orbit is to be preferred. The two old sets of velocities have such low weights that they do not significantly improve the other elements. Thus, with the period fixed from the previous circular-orbit solution and two NSO velocities given zero weight because of their large residuals, a final circular orbit was computed with only the NSO and KPNO data. Those orbital elements are listed in Table 5. Since the eccentricity is zero, the time of periastron is undefined and has been replaced with the time of maximum positive velocity.

Our velocities and residuals to the computed fit are given in Table A5 in the electronic Appendix. The computed radial-velocity curve is compared with our velocities in Fig. 4. The standard error of an observation of unit weight is $0.6 \mathrm{~km} \mathrm{~s}^{-1}$, quite good for such a broad lined star.

\section{HD $33363=$ CL Cam}

\subsection{Brief history}

HD 33363 was added to the second edition of the CABS catalog on the basis of its strong CaII $\mathrm{H}$ and $\mathrm{K}$ emission (Bidelman 1991; Strassmeier 1994) and its variable velocity. Henry et al. (1995) classified the star as K0 III, noted a preliminary orbital period of 20.9 days, and found a period of 41.5 days for its photometric variability. Such disparate periods are unusual but not unknown among active binaries. In fact, the orbital and rotational periods found for HD 33363 are similar to those of the well-known asynchronous rotator $\lambda$ And.

\subsection{Orbital elements}

Between 1991 and 1998 two radial-velocity data sets were obtained at the KPNO for HD 33363; a more extensive set of 28 velocities by FCF and 10 velocities in January 1996 by the Vienna group. A preliminary set of orbital elements was determined for the FCF velocities with BISP. After one velocity was given zero weight, those elements were refined with SB1. The Vienna group's velocities were compared with this orbit. As a result, no zero-point correction was applied to the January 1996 velocities, and those individual velocities were given a weight of 0.05 in the final orbital solution, whose elements are listed in Table 5 . The individual velocities and residuals to the computed fit are given in Table A6 in the Appendix. Shown in Fig. 5 is the computed velocity curve compared with our velocities.

We note that for the final solution the standard error of an observation of unit weight is $0.15 \mathrm{~km} \mathrm{~s}^{-1}$, the best so far achieved with the coudé feed telescope for an orbit of a chromospherically active star. Since the orbit has a small but definite eccentricity of $0.071 \pm 0.003$, HD 33363 is still in the process of both circularization and synchronization. A time of conjunction with the primary behind the secondary is HJD 2449311.371. 


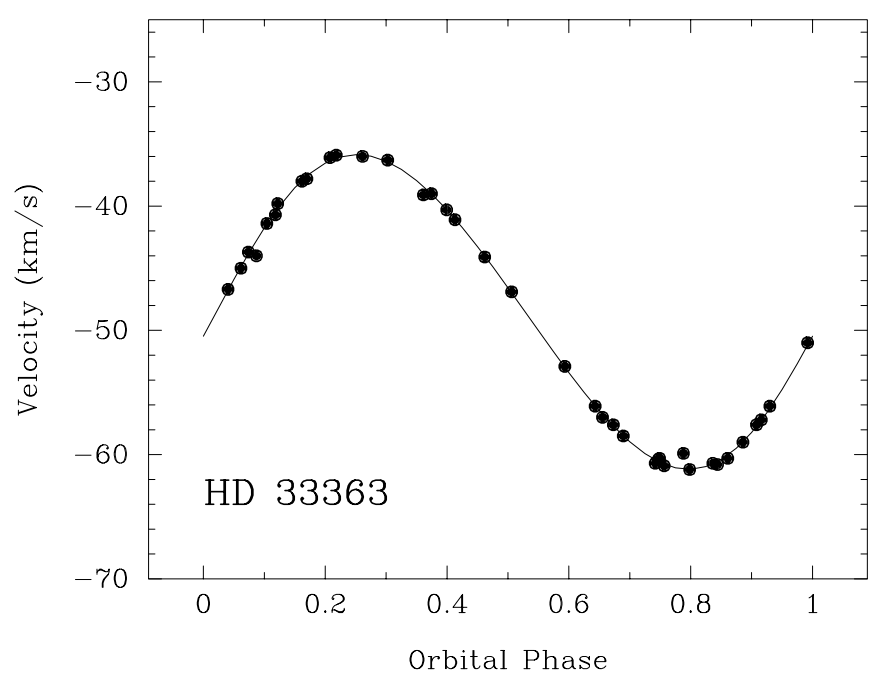

Fig. 5. Radial-velocity curve of HD 33363. All data were obtained at KPNO

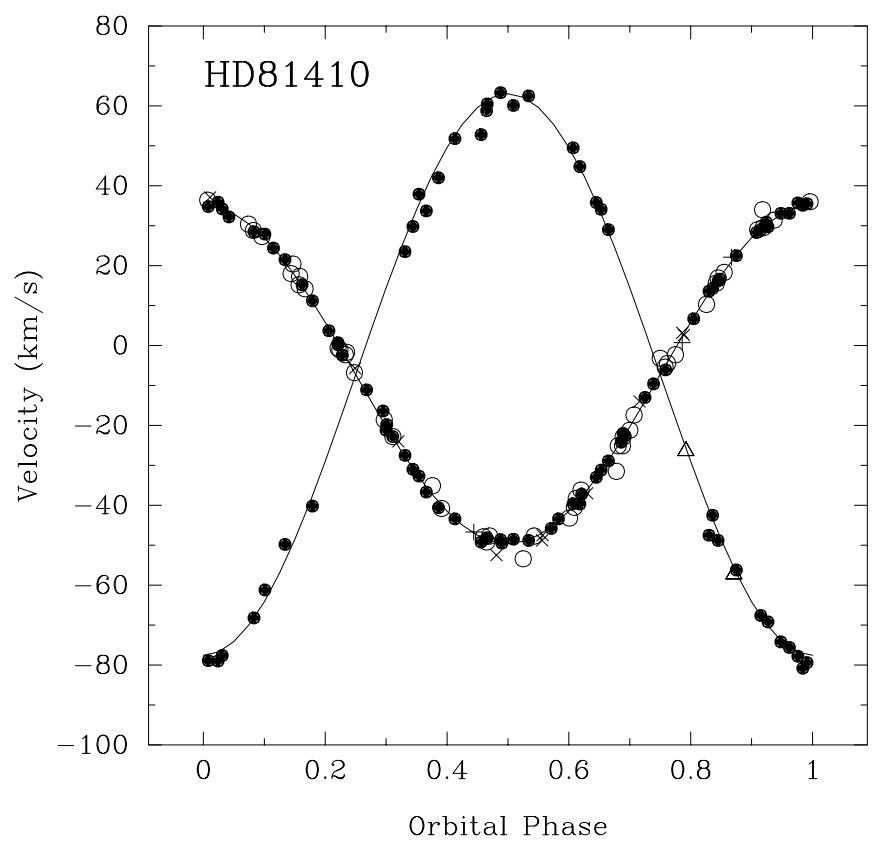

Fig. 6. Radial-velocity curves for HD 81410 . Filled circles are from KPNO, open circles from NSO, pluses from McDonald. Additionally, we added ten velocities from Dadonas (1994) (crosses) and two velocities of both the primary and secondary from Donati et al. (1997) (triangles). Zero phase is the time of maximum positive velocity of the primary

\section{HD81410 = IL Hya}

\subsection{Brief history}

The light varibility of HD 81410, suspected by Cousins \& Stoy (1963), was confirmed by Eggen (1973), who found a $V$ amplitude of 0.4 and a period of 25.4 days. From additional photometry Raveendran et al. (1982) discovered that a period of 12.86833 satisfied all available photometry and identified the star as an RS CVn binary. Combining
25 years of $V$-band photometry, Strassmeier et al. (1997) obtained a long-term average period of 12.7864 days.

Bidelman \& MacConnell (1973) classified HD 81410 as K1 III and noted that it has strong Ca II H and K emission and also that its Balmer lines are filled in. Radial velocity observations of Wayman \& Jones (Eggen 1973) showed the star to be a single-lined binary, and Raveendran et al. (1982) found the velocities to be consistent with their photometric period. From spectroscopic and photometric evidence they suggested that the secondary is probably an F dwarf. Balona (1987) combined his numerous velocities with those of Collier Cameron (1987) and determined an orbital period of 12.908 days. Following the spectroscopic discovery of the secondary (Donati et al. 1997), Weber \& Strassmeier (1998) obtained multiwavelength Doppler images of HD 81410 and computed the first double-lined spectroscopic orbit for it. Recently, using 85 published velocities, Raveendran \& Mekkaden (1998) determined both circular and elliptical orbits for the primary.

\subsection{Orbital elements}

We have obtained 101 observations of HD 81410 from 1981 through 1998. Of that total, 31 observations were made by FCF at McDonald Observatory and KPNO and 80 by the Vienna group at NSO and KPNO. Some of the velocities have previously appeared in Fekel et al. (1986) and Weber \& Strassmeier (1998). The values used in this paper are slightly different due to the assumption of different standard-star velocities. In addition the Vienna group's KPNO H $\alpha$ spectra of February-March 1995 have been remeasured.

Complicating the orbital analysis of this star is the primary's significant chromospheric activity, resulting in line profile variability, plus the fact that 10 different data sets, containing from two to 46 velocities and totaling 179 velocities, are available for the primary.

Preliminary orbital elements of the primary were computed initially for the FCF velocities with BISP and then refined with SB1. Similar independent orbital solutions were obtained for the velocities of Balona (1987), the Vienna group's NSO velocities, and the Vienna group's KPNO velocities obtained at $6430 \AA$, to determine the weights of the observations and any zero-point velocity shift for each data set.

The other six data sets contain only a very small number of velocities or do not have good phase coverage. Since the FCF velocities have the longest baseline, from 1981 to 1998 , of any of the data sets, the orbital solution of those velocities provides an excellent "template" for the other six data sets. Thus, the six sets of velocities were included with zero weight in a separate SB1 solution of the FCF velocities. The velocity residuals to the FCF-velocity solution for each of the six data sets were used to determine the appropriate weights and zero-point shifts. The results 
Table 4. HD 81410 primary data sets

\begin{tabular}{llrlc}
\hline Data Set & Years & No. & Wt. & $\begin{array}{c}\Delta v_{0} \\
\left(\mathrm{~km} \mathrm{~s}^{-1}\right)\end{array}$ \\
\hline Wayman (Eggen 1973) & 1959 & 4 & 0.01 & 0.0 \\
Jones \& Fisher (1984) & 1972 & 3 & 0.1 & 0.0 \\
Balona (1987) & $1979-1981$ & 34 & 0.1 & 0.0 \\
Collier-Cameron (1987) & 1981 & 15 & 0.05 & 0.0 \\
FCF (this paper) & $1981-1998$ & 31 & 1.0 & 0.0 \\
Dadonas (this paper) & 1993 & 10 & 0.2 & 1.9 \\
Donati et al. (1997) & 1993 & 2 & 1.0 & 0.0 \\
Vienna group KPNO (this paper) & $1994-1998$ & 25 & 1.0 & 1.2 \\
Vienna group KPNO H $\alpha$ (this paper) & 1995 & 9 & 1.0 & 0.0 \\
Vienna group NSO (this paper) & $1996-1997$ & 46 & 0.2 & 1.7 \\
\hline
\end{tabular}

for all 10 data sets are summarized in Table 4, where Col. 1 identifies the data set, Col. 2 notes the years covered, and Col. 3 gives the number of velocities. Columns 4 and 5 list the weight assigned to the velocities in each set and each set's zero-point shift, respectively.

An SB1 orbital solution of all the appropriately weighted velocities of the primary resulted in an eccentricity of $0.0046 \pm 0.0027$. Because of this very low eccentricity, a circular-orbit solution for the same data was computed with SB1C. According to the precepts of Lucy \& Sweeney (1971), the circular orbit is to be preferred.

Very weak lines of the secondary are visible in 35 of our spectra that have the highest signal-to-noise ratios. Independent SB1 solutions, assuming the elements of the primary except for the center-of-mass velocity and semiamplitude, were obtained for the FCF velocities and the Vienna group's KPNO velocities. Those 35 secondary velocities plus two of Donati et al. (1997) were given a weight of 0.1 and no zero-point correction was applied.

An all-data circular orbit for the primary and secondary velocities together was computed with SB2C. Six velocities of the primary and one of the secondary were given zero weight because of their large residuals. The period from that solution was assumed, and a final SB2C solution was obtained with all velocities having weights $\geq 0.1$. Those orbital elements are listed in Table 5. Since the eccentricity is zero, the time of periastron is undefined and has been replaced with the time of maximum positive velocity.

Table A7 in the Appendix lists our velocities of the primary, those of Donati et al. (1997) as well as those obtained by Dadonas (1994), at Maidanak, Uzbekistan (Dadonas et al. 1994). Table A8 in the Appendix gives our velocities of the secondary and the two values of Donati et al. (1997). Figure 6 shows the computed velocity curves compared to the velocities listed in our two tables. The standard error of an observation of unit weight for the primary is $0.7 \mathrm{~km} \mathrm{~s}^{-1}$, quite good for such an active star.

\section{HD $106225=$ HU Vir}

\subsection{Brief history}

HD 106225 was transformed from an obscure eighth magnitude star into a stellar source of significant notoriety after Bidelman (1981) noted that this late-type star has strong Ca II $\mathrm{H}$ and $\mathrm{K}$ emission. Following its discovery as a chromospherically active star, Fekel et al. (1984) obtained spectroscopic and photometric observations of it. They determined a preliminary spectroscopic period of 10.389 days and preferred a photometric period of 10.6 days with a $V$ amplitude of 0.25 . Recently, Strassmeier et al. (1997) found a long-term average photometric period of 10.418 days from 14 years of $V$-band photometry, which is closer to the orbital period.

Fekel et al. (1986) found that the $\mathrm{H} \alpha$ line of HD 106225 was slightly in emission above the continuum, while Strassmeier et al. (1990) obtained a spectrum showing $\mathrm{H} \epsilon$ emission in addition to strong $\mathrm{Ca}$ II $\mathrm{H}$ and $\mathrm{K}$ emission. Such $\mathrm{H} \alpha$ and $\mathrm{H} \epsilon$ emission is seen in only the most active RS CVn binaries. Strassmeier (1994b) found very significant absorption-line variations and used numerous spectroscopic and photometric observations of HD 106225 to produce two 1991 Doppler images of its surface-temperature distribution. He also obtained improved orbital elements for the binary.

\subsection{Orbital elements}

From 1981 through 1998, 178 observations were obtained of HD 106225. Of that total, 66 were made by FCF at McDonald Observatory and KPNO and 112 by the Vienna group at NSO and KPNO. Some of those velocities have been previously published by Fekel et al. (1984) and Strassmeier (1994b). The values used in this paper are slightly different due to the assumption of different standard-star velocities.

Determination of the orbital elements is complicated by the star's strong chromospheric activity, resulting in 

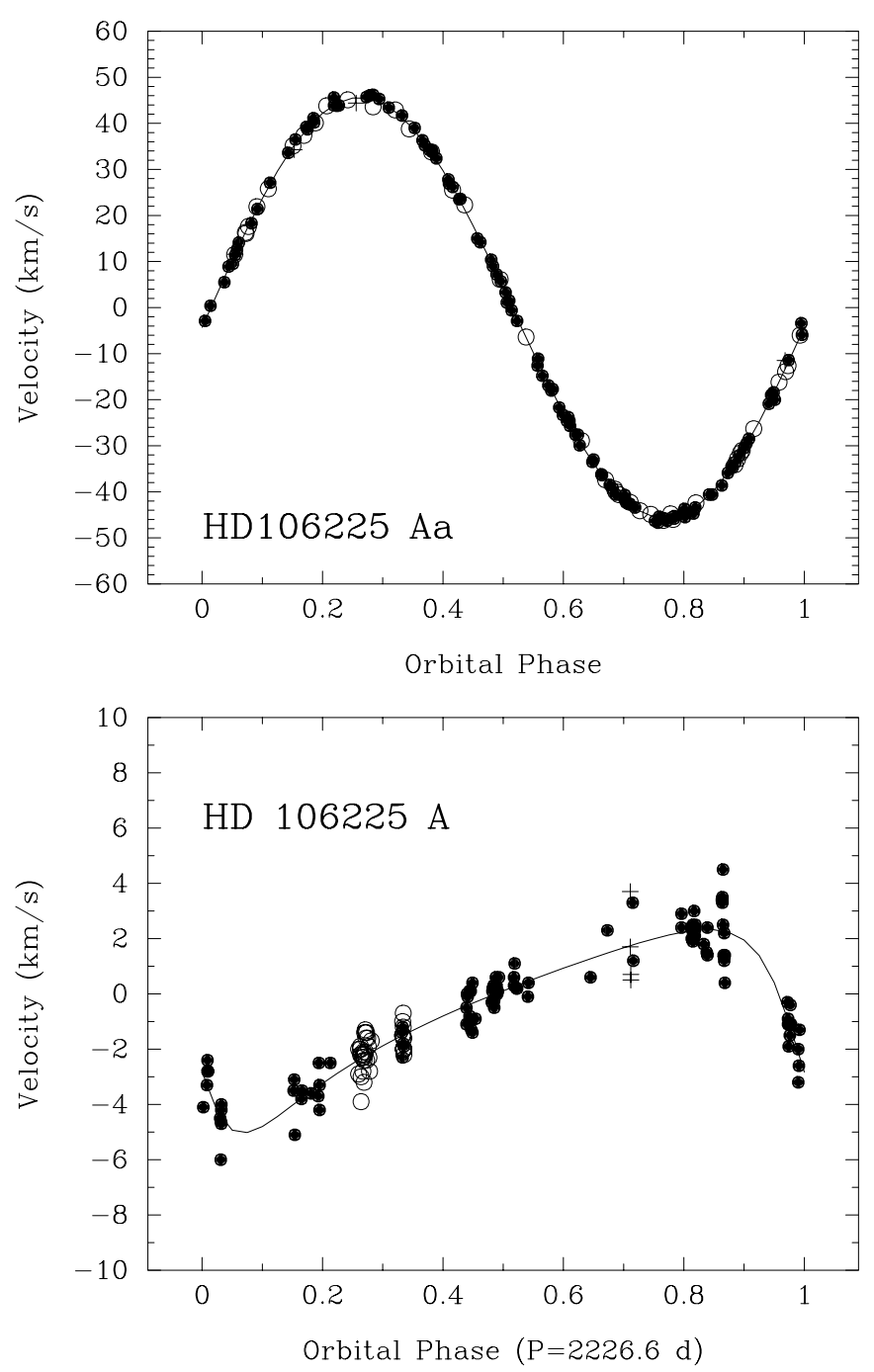

Fig. 7. Radial-velocity curves of HD $106225=$ HU Vir. The upper panel shows the short-period orbit of Aa, the lower panel, the long-period orbit of A. Open symbols are NSO McMathPierce data, filled symbols are from KPNO, pluses are from McDonald Observatory

significant line-profile variability, and the discovery that the system is triple.

Assuming the orbital elements of Strassmeier (1994b) as starting values, a set of orbital elements for the FCF velocities was determined with SB1. The velocity residuals of that solution showed systematic variations indicating the presence of a third component. A long period of about 6.3 years was identified from a period search done on the residuals. Preliminary long-period elements for the residuals were computed with BISP. Then a simultaneous solution of the short- and long-period orbits was obtained with GLS.

Although the velocities of the Vienna group are more numerous, the seven sets of spectra were obtained primarily for spot modelling, and so the velocities are not well distributed in phase in the long-period orbit. To determine possible velocity offsets, the velocities obtained by the Vienna group were included with zero weight in a GLS solution of the FCF velocities. A mean residual was computed for each of the Vienna group's seven sets of velocities. As a result, velocity offsets were applied to three of the data sets, $-1.3 \mathrm{~km} \mathrm{~s}^{-1}$ for the April 1991 NSO data, $+1.0 \mathrm{~km} \mathrm{~s}^{-1}$ for the March $1994 \mathrm{KPNO}$ velocities, and $+2.0 \mathrm{~km} \mathrm{~s}^{-1}$ for the February $1995 \mathrm{KPNO}$ velocities. No velocity shift was applied to the three most recent velocity sets. Velocity residuals for the April 1991 KPNO velocities ranged from +6 to $-10 \mathrm{~km} \mathrm{~s}^{-1}$, and so, velocities in that data set were not included in the final solution.

Elements for the short- and long-period orbits, computed from a GLS solution that included 154 velocities, are given in Table 5. The short-period eccentricity is quite small $0.0093 \pm 0.0033$, but has been retained because in triple systems the third star may cause a non-zero eccentricity (Mazeh \& Shaham 1979; Söderhjelm 1984) in the short-period orbit.

Although only one component shows both long- and short-period orbital motion, for the purposes of the tables and figures we identify the long-period orbit as component $\mathrm{A}$ and the submotion or short-period orbit as Aa. The short-period velocities and residuals to the computed fit are listed in Table A9 in the Appendix, while those for the long-period are in Table A10. For the tables and figures each measured velocity has been separated into a short- and long-period component, which consists of the short- or long-period calculated velocity plus one-half of the computed residual. Thus, the sum of those separated short- and long-period velocities for each date of observation results in the actually measured velocity. The computed short-period velocity curve compared with our velocities is shown in the upper panel of Fig. 7, while the long-period velocity curve is shown in the lower panel of Fig. 7. The standard error of an observation of unit weight is $1.3 \mathrm{~km} \mathrm{~s}^{-1}$, primarily the result of the broad and asymmetric lines of this star. A time of conjunction in the short-period orbit with the primary behind the secondary is HJD 2450195.909.

\section{HD $136901=$ UV CrB}

\subsection{Brief history}

Heard (1956) discovered the velocity variability of HD 136901 from nine radial velocities obtained at David Dunlap Observatory. A hiatus of several decades ensued before interest in this star was revived with Bidelman's (1983) discovery of weak CaII $\mathrm{H}$ and $\mathrm{K}$ emission in an objective-prism spectrum of HD 136901. That result spurred additional spectroscopic and photometric analyses. Fekel et al. (1986) obtained a preliminary spectroscopic period of 18.68 days, nearly twice the 


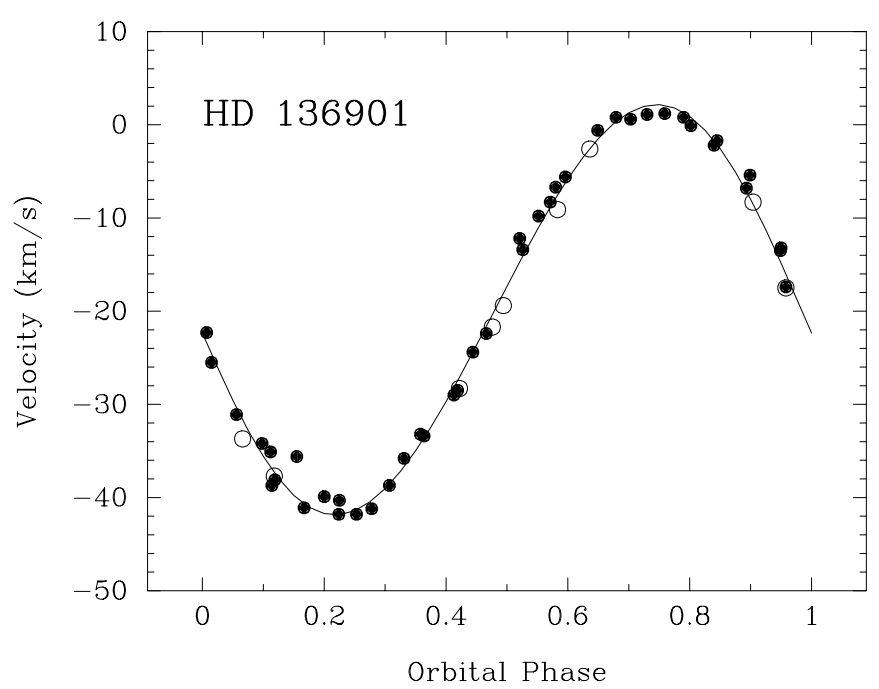

Fig. 8. Radial-velocity curve of HD 136901. Data were obtained at KPNO (dots) and NSO (open circles) and were given equal weight except one KPNO velocity at phase 0.16 that was given zero weight

photometric period of 9.63 days discovered by Boyd et al. (1984). Fekel et al. (1989) published a full set of orbital elements and discussed the properties of the system. Strassmeier et al. (1989) confirmed the suggestion of Fekel et al. (1986) that the light variations are mainly due to the ellipticity effect. Kaye et al. (1995) evaluated a number of seasons of photometry of HD 136901 and removed the ellipticity effect. The remaining residuals were analyzed and in seven of the 11 data sets showed a periodicity within a few percent of the orbital period. Those additional light variations are presumably due to starspots.

\subsection{Orbital elements}

The Vienna group obtained 18 new velocities, nine at NSO and nine at KPNO. Those velocities were combined with two other previously published data sets, those of Fekel et al. (1989) and Heard (1956). Because of different assumed standard-star velocities, the Fekel et al. (1989) velocities used in the present analysis differ slightly from the originally published values.

We first computed independent SB1 orbital solutions of the velocities of Fekel et al. (1989) and of the combined set of velocities of the Vienna group. A comparison of the two solutions resulted in no zero-point shift to any of the data sets and unit weight assigned to all velocities, except for one velocity given zero weight. With Heard's (1956) velocities given weights of 0.01 and adjusted by $-4 \mathrm{~km} \mathrm{~s}^{-1}$, an orbital solution of all the data was obtained. The period was fixed at that value, and an orbital solution with SB1 was obtained for the revised Fekel et al. (1989) velocities plus those of the Vienna-group. The eccentricity of the orbit, $0.045 \pm 0.011$, although reduced slightly from the

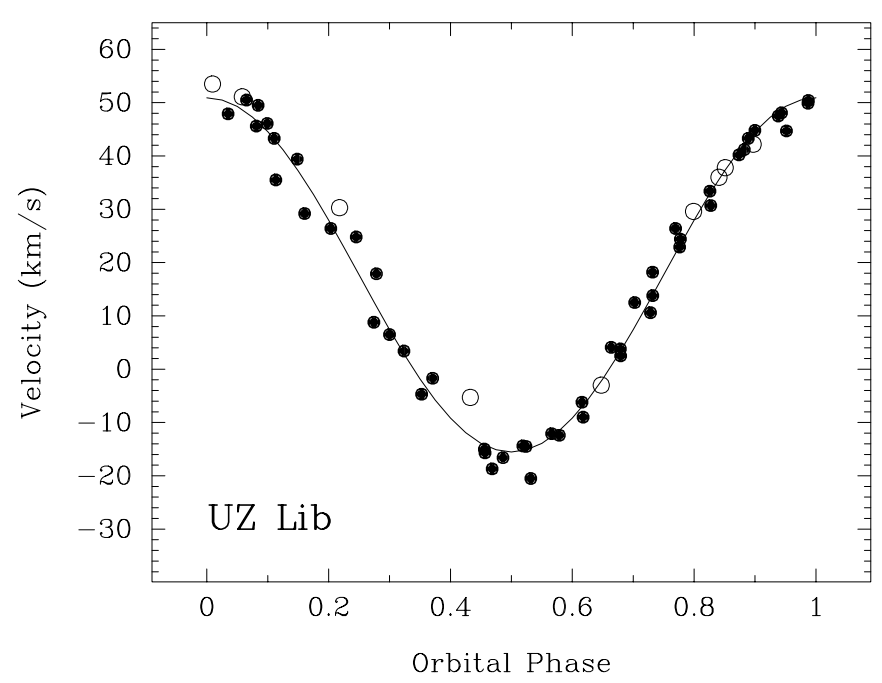

Fig. 9. Radial-velocity curve of UZ Lib. Data were obtained at KPNO (filled circles) and ESO (open circles) and were given equal weight except for one velocity at phase 0.43 that was given zero weight

value of $0.059 \pm 0.014$ obtained by Fekel et al. (1989), is still assumed to be real, in accordance with the precepts of Lucy \& Sweeney (1971). Table 5 lists this eccentric-orbit solution. The individual observations and velocity residuals are presented in Table A11 in the Appendix. The calculated velocity curve is compared with the observed velocities in Fig. 8.

The standard error of an observation of unit weight is $1.2 \mathrm{~km} \mathrm{~s}^{-1}$, primarily the result of the very broad lines of this star. A time of conjunction with the primary behind the secondary is HJD 2447312.447.

\section{1. $\mathrm{BD}-08^{\circ} 3999=\mathrm{UZ}$ Lib}

\subsection{Brief history}

UZ Lib is a star whose true nature was misinterpreted for several decades. Over 60 years ago Parenago (1931) found it to have light variations and concluded that it was an RR Lyrae variable. From new photometry Wiesniewski (1973) determined a period of 9.5 days and classified it as a $\beta$ Lyrae-type system. Evans \& Bopp (1974) proposed that UZ Lib might be a spotted-dwarf flare star, a suggestion that ultimately proved to be close to the mark. Hoffmann (1980) obtained additional photometry and found it consistent with a period of 4.75 days. Bopp et al. (1981) confirmed the likelyhood of the 4.75 day photometric period and determined a $V$-band amplitude of 0.35 . Recently, from $V$-band photometry spanning 25 years Strassmeier et al. (1997) obtained a long-term average photometric period of 4.75226 days.

Bidelman (1954) reported the discovery of its Ca II H and $\mathrm{K}$ emission, gave its spectral type as $\mathrm{Kp}$, and listed it in a table of spectroscopic binaries, although no reference 
for its duplicity was given. Bopp \& Stencel (1981) proposed that UZ Lib is an FK Comae-type star. This class of photometric variables consists of a very small group of chromospherically active single late-type giants that have extremely rapid rotation. The radial velocities of Woolley et al. (1981), however, indicated that the star is a binary. Bopp et al. (1984) obtained additional velocities and determined an orbital period of 4.7678 days.

\subsection{Orbital elements}

Two new sets of velocities were obtained by the Vienna group. From March 1994 through April 1998, 49 observations were made at KPNO, while 9 additional velocities were obtained in May 1996 at ESO. Velocities of the March 1994 spectra have previously been published by Strassmeier (1996). Our velocities for those spectra are slightly different because of different assumed velocities for the standard stars.

For our KPNO data an initial set of orbital elements was determined with BISP and refined with SB1. Then the ESO velocities, given unit weight, were included in a new orbital solution. In a similar manner independent orbital solutions were determined for the Woolley et al. (1981) and the Bopp et al. (1984) velocities. From the resulting variance of the data set and the center-of-mass velocity, the Woolley et al. (1981) data were given weights of 0.2 , and $10.0 \mathrm{~km} \mathrm{~s}^{-1}$ was added to each velocity. Results for the Bopp et al. (1984) velocities were 0.25 and $-2.0 \mathrm{~km} \mathrm{~s}^{-1}$. Because of their small number and uncertain velocity offset, the velocities of Grewing et al. (1989) were not included in our analysis.

An SB1 solution with the four velocity sets appropriately weighted, except for three velocities given zero weight, was compared to a solution of our new velocities that had the period fixed from the all-data solution. Since only the error of the period was improved in the all-data solution, the solution of our new velocities, having an eccentricity of $0.041 \pm 0.016$, was compared with a circular-orbit solution. Although a close call, according to the precepts of Lucy \& Sweeney (1971), a circular orbit is to be prefered. Thus, Table 5 lists the orbital elements of UZ Lib, determined with the SB1C program and our velocities only (one ESO velocity was given zero weight) and with the period fixed from the all-data circular solution. Since the orbit is circular, a time of maximum positive velocity is listed rather than a time of periastron passage. Table A12 in the Appendix lists our velocity observations and the residuals to the computed fit. Shown in Fig. 9 is the computed orbit compared with our velocities. The combination of very substantial rotational broadening and line profile asymmetries results in a standard error of $3.0 \mathrm{~km} \mathrm{~s}^{-1}$ for an observation of unit weight.

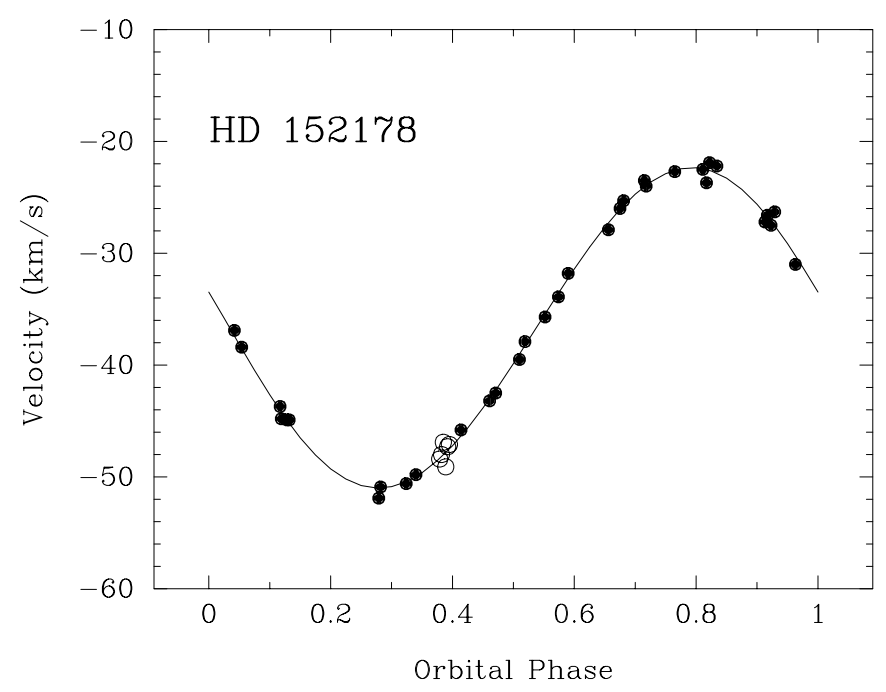

Fig. 10. Radial-velocity curve of HD 152178. Data obtained at KPNO and ESO are shown as filled and open circles, respectively, and were given equal weight

\section{HD $152178=$ V2253 Oph}

\subsection{Brief history}

HD 152178 was classified as G8/K0Vp and found to have Ca II H and $\mathrm{K}$ emission by Houk (1982). However, our spectra indicate that the star is a K0 III (Strassmeier et al. 1993), a result consistent with its $M_{V}$ determined from its Hipparcos parallax (ESA 1997). Hooten \& Hall (1990) found a photometric period of 22.35 days. Our preliminary orbital elements were listed in the second edition of the CABS catalog (Strassmeier et al. 1993). The orbital period of 314.5 days is much longer than the rotational period, making the star an asynchronous rotator.

\subsection{Orbital elements}

Between 1986 and 1996 FCF obtained 32 observations at KPNO. The Vienna group made an additional 6 observations during an observing run in May 1996 at ESO plus a single observation at KPNO in 1998.

A preliminary orbital solution for the data of FCF was obtained with BISP, and those elements were refined with SB1. Then, the seven additional velocities were compared with this orbit. As a result, an orbital solution was computed with all 39 observations given unit weight, and no zero-point correction applied to any of the data. Because the eccentricity of that solution is quite small, $0.0236 \pm 0.0091, \mathrm{SB} 1 \mathrm{C}$ was used to compute a circular orbit with the full set of data. Following the precepts of Lucy \& Sweeney (1971), the eccentric-orbit solution has been retained, and those orbital elements are listed in Table 5. Our radial velocities and velocity residuals to the computed orbit are listed in Table A13 in the Appendix. 
Table 5. Orbital elements

\begin{tabular}{|c|c|c|c|c|c|c|c|c|c|}
\hline Star & $\begin{array}{l}P_{\text {orb }} \\
(\text { days })\end{array}$ & $\begin{array}{l}T_{0} \\
(244+)\end{array}$ & $\begin{array}{l}T_{\text {Peri }} \\
(244+)\end{array}$ & $\begin{array}{l}v_{0} \\
\left(\mathrm{~km} \mathrm{~s}^{-1}\right)\end{array}$ & $\begin{array}{l}K \\
\left(\mathrm{~km} \mathrm{~s}^{-1}\right)\end{array}$ & $e$ & $\begin{array}{l}\omega \\
\left({ }^{\circ}\right)\end{array}$ & $\begin{array}{l}a \sin i^{a} \\
\left(10^{6} \mathrm{~km}\right)\end{array}$ & $\begin{array}{l}f(m)^{b} \\
\left(M_{\odot}\right)\end{array}$ \\
\hline LN Peg A & $\begin{array}{l}1564.4 \\
\pm 12.8\end{array}$ & $\ldots$ & $\begin{array}{l}8396.5 \\
\pm 392.8\end{array}$ & $\begin{array}{l}-15.30 \\
\pm 0.30\end{array}$ & $\begin{array}{l}6.39 \\
\pm 0.67\end{array}$ & $\begin{array}{l}0.054 \\
\pm 0.064\end{array}$ & $\begin{array}{l}146.4 \\
\pm 89.8\end{array}$ & $\begin{array}{l}137.2 \\
\pm 6.7\end{array}$ & $\begin{array}{l}0.0421 \\
\pm 0.0062\end{array}$ \\
\hline LN Peg Aa & $\begin{array}{l}1.8441701 \\
\pm 0.0000012\end{array}$ & & $\begin{array}{l}6347.832 \\
\pm 0.508\end{array}$ & & $\begin{array}{l}62.38 \\
\pm 0.25\end{array}$ & $\begin{array}{l}0.0025 \\
\pm 0.0042\end{array}$ & $\begin{array}{l}41.4 \\
\pm 99.3\end{array}$ & $\begin{array}{l}1.5819 \\
\pm 0.0066\end{array}$ & $\begin{array}{l}0.4052 \\
\pm 0.0096\end{array}$ \\
\hline LN Peg Ab & & & & & $\begin{array}{l}90.51 \\
\pm 0.99\end{array}$ & & & $\begin{array}{l}2.295 \\
\pm 0.035\end{array}$ & $\begin{array}{l}0.2792 \\
\pm 0.0052\end{array}$ \\
\hline$\zeta$ And & $\begin{array}{l}17.769426 \\
\pm 0.000040\end{array}$ & $\begin{array}{l}9992.781 \\
\pm 0.017\end{array}$ & $\ldots$ & $\begin{array}{l}-24.43 \\
\pm 0.11\end{array}$ & $\begin{array}{l}25.11 \\
\pm 0.15\end{array}$ & 0.0 & $\ldots$ & $\begin{array}{l}6.14 \\
\pm 0.36\end{array}$ & $\begin{array}{l}0.02923 \\
\pm 0.00052\end{array}$ \\
\hline HD 33363 & $\begin{array}{l}20.86932 \\
\pm 0.00043\end{array}$ & $\ldots$ & $\begin{array}{l}9306.111 \\
\pm 0.175\end{array}$ & $\begin{array}{l}-48.378 \\
\pm 0.036\end{array}$ & $\begin{array}{l}12.661 \\
\pm 0.041\end{array}$ & $\begin{array}{l}0.0712 \\
\pm 0.0033\end{array}$ & $\begin{array}{l}261.1 \\
\pm 3.1\end{array}$ & $\begin{array}{l}3.624 \\
\pm 0.012\end{array}$ & $\begin{array}{l}0.004366 \\
\pm 0.000042\end{array}$ \\
\hline HD 81410 A & $\begin{array}{l}12.904982 \\
\pm 0.000040\end{array}$ & $\begin{array}{l}7093.4936 \\
\pm 0.0054\end{array}$ & $\ldots$ & $\begin{array}{l}-7.274 \\
\pm 0.080\end{array}$ & $\begin{array}{l}42.29 \\
\pm 0.12\end{array}$ & 0.0 & $\ldots$ & $\begin{array}{l}7.505 \\
\pm 0.021\end{array}$ & $\begin{array}{l}1.194 \\
\pm 0.019\end{array}$ \\
\hline HD $81410 \mathrm{~B}$ & & & & & $\begin{array}{l}70.30 \\
\pm 0.48\end{array}$ & & & $\begin{array}{l}12.475 \\
\pm 0.086\end{array}$ & $\begin{array}{l}0.7184 \\
\pm 0.0071\end{array}$ \\
\hline HD $106225 \mathrm{~A}$ & $\begin{array}{l}2226.6 \\
\pm 12.1\end{array}$ & $\ldots$ & $\begin{array}{l}9833.4 \\
\pm 30.8\end{array}$ & $\begin{array}{l}-0.66 \\
\pm 0.12\end{array}$ & $\begin{array}{l}3.68 \\
\pm 0.29\end{array}$ & $\begin{array}{l}0.474 \\
\pm 0.051\end{array}$ & $\begin{array}{l}113.5 \\
\pm 7.8\end{array}$ & $\begin{array}{l}99.3 \\
\pm 8.0\end{array}$ & $\begin{array}{l}0.0079 \\
\pm 0.0020\end{array}$ \\
\hline HD $106225 \mathrm{Aa}$ & $\begin{array}{l}10.387552 \\
\pm 0.000031\end{array}$ & $\ldots$ & $\begin{array}{l}9993.195 \\
\pm 0.602\end{array}$ & $\ldots$ & $\begin{array}{l}45.66 \\
\pm 0.16\end{array}$ & $\begin{array}{l}0.0093 \\
\pm 0.0033\end{array}$ & $\begin{array}{l}264.7 \\
\pm 20.9\end{array}$ & $\begin{array}{l}6.522 \\
\pm 0.024\end{array}$ & $\begin{array}{l}0.1027 \\
\pm 0.0013\end{array}$ \\
\hline HD 136901 & $\begin{array}{l}18.66692 \\
\pm 0.00031\end{array}$ & . & $\begin{array}{l}7312.757 \\
\pm 0.750\end{array}$ & $\begin{array}{l}-19.73 \\
\pm 0.18\end{array}$ & $\begin{array}{l}22.01 \\
\pm 0.25\end{array}$ & $\begin{array}{l}0.045 \\
\pm 0.011\end{array}$ & $\begin{array}{l}96.5 \\
\pm 14.5\end{array}$ & $\begin{array}{l}5.645 \\
\pm 0.064\end{array}$ & $\begin{array}{l}0.02062 \\
\pm 0.00070\end{array}$ \\
\hline UZ Lib & $\begin{array}{l}4.768241 \\
\pm 0.000026\end{array}$ & $\begin{array}{l}9993.662 \\
\pm 0.013\end{array}$ & $\ldots$ & $\begin{array}{l}17.69 \\
\pm 0.40\end{array}$ & $\begin{array}{l}33.22 \\
\pm 0.55\end{array}$ & 0.0 & $\ldots$ & $\begin{array}{l}2.178 \\
\pm 0.036\end{array}$ & $\begin{array}{l}0.01815 \\
\pm 0.00091\end{array}$ \\
\hline HD 152178 & $\begin{array}{l}314.47 \\
\pm 0.18\end{array}$ & $\ldots$ & $\begin{array}{l}8215.6 \\
\pm 29.6\end{array}$ & $\begin{array}{l}-36.75 \\
\pm 0.13\end{array}$ & $\begin{array}{l}14.32 \\
\pm 0.15\end{array}$ & $\begin{array}{l}0.0236 \\
\pm 0.0091\end{array}$ & $\begin{array}{l}77.1 \\
\pm 34.1\end{array}$ & $\begin{array}{l}61.92 \\
\pm 0.64\end{array}$ & $\begin{array}{l}0.0959 \\
\pm 0.0030\end{array}$ \\
\hline HD 208472 & $\begin{array}{l}22.62293 \\
\pm 0.00097\end{array}$ & $\begin{array}{l}9252.391 \\
\pm 0.038\end{array}$ & $\ldots$ & $\begin{array}{l}10.160 \\
\pm 0.081\end{array}$ & $\begin{array}{l}16.24 \\
\pm 0.12\end{array}$ & 0.0 & $\ldots$ & $\begin{array}{l}5.051 \\
\pm 0.036\end{array}$ & $\begin{array}{l}0.01006 \\
\pm 0.00022\end{array}$ \\
\hline HD 216489 & $\begin{array}{l}24.648944 \\
\pm 0.000075\end{array}$ & $\begin{array}{l}9227.690 \\
\pm 0.022\end{array}$ & $\ldots$ & $\begin{array}{l}-15.47 \\
\pm 0.13\end{array}$ & $\begin{array}{l}34.30 \\
\pm 0.17\end{array}$ & 0.0 & $\ldots$ & $\begin{array}{l}11.625 \\
\pm 0.058\end{array}$ & $\begin{array}{l}0.1033 \\
\pm 0.0015\end{array}$ \\
\hline
\end{tabular}

${ }^{a}$ If single lined, $a_{1} \sin i$; if double lined, both semi-major axes are given.

${ }^{b}$ If double lined, $m \sin ^{3} i$.

The computed orbit is compared to the observed velocities in Fig. 10. The standard error of an observation of unit weight is $0.6 \mathrm{~km} \mathrm{~s}^{-1}$. A time of conjunction with the primary behind the secondary is HJD 2448226.377.

HD 152178 is clearly an asynchronous rotator and an interesting system but we note that the long orbital period suggests only weak tidal forces to synchronize the rotation.

\section{HD $208472=$ V2075 Cyg}

\subsection{Brief history}

HD 208472 was identified as a chromospherically active star by Bidelman (1991), who discovered its CaII H and K emission features. Henry et al. (1995) found photometric variations with a period of 22.54 days and determined a preliminary orbital period of 22.6 days, making this star a synchronous rotator. They concluded that the system is very active as evidenced by its large-amplitude $V$-band variations of 0.36 and an $\mathrm{H} \alpha$ line above the continuum.

\subsection{Orbital elements}

From 1992 through 1998, 47 velocities were obtained at KPNO, 43 by FCF and 4 by the Vienna group. An additional 51 velocities were gathered by the Vienna group at NSO during a single 1996-1997 observing run.

Because of the much longer timespan of the data, orbital elements for the KPNO velocities were computed first with BISP, and refined with SB1. Then, an orbital solution for the NSO velocities was obtained, assuming the period from the KPNO-velocity solution. From a comparison of the two solutions, each NSO velocity was assigned a weight of 0.15 relative to the KPNO velocities, and no zero-point velocity correction was made. An orbital solution with the appropriately weighted data resulted in $e=0.0058 \pm 0.0071$, and so a circular-orbit solution was computed with SB1C. According to the precepts of Lucy \& Sweeney (1971), the circular orbit is clearly preferable, and those elements are given in Table 5. Since the eccentricity is zero, the time of periastron passage has been replaced by the time of maximum velocity. Figure $11 \mathrm{com}-$ pares the computed orbit with the observed velocities, while our radial velocities and velocity residuals to the 


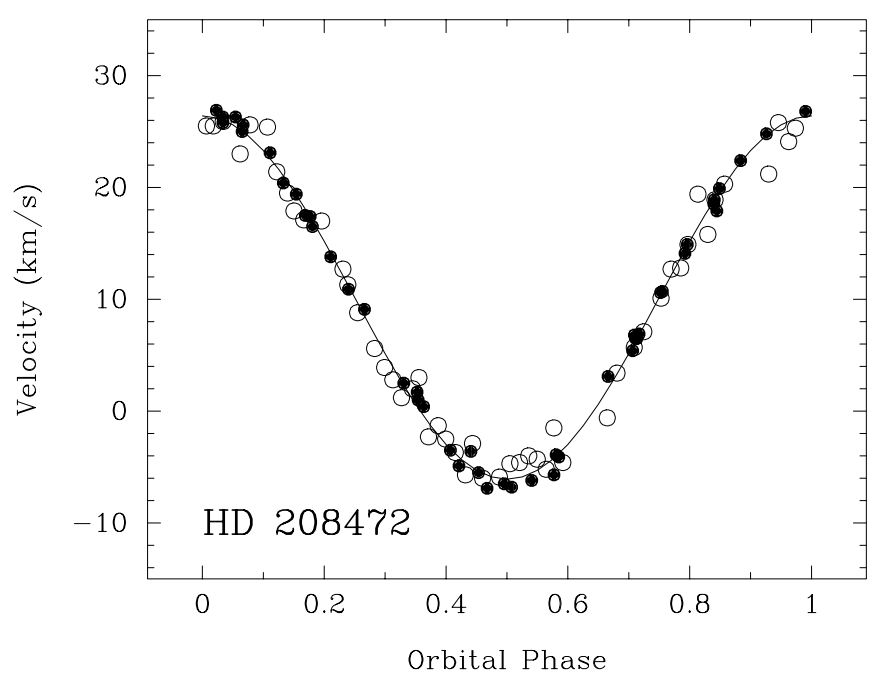

Fig. 11. Radial-velocity curve of HD 208472. Filled circles are data obtained at KPNO and open circles are data obtained at NSO. The latter had inferior spectral coverage compared to the KPNO data and were given a weight of 0.15

computed orbit are given in Table A14 in the Appendix. The standard error of an observation of unit weight is $0.6 \mathrm{~km} \mathrm{~s}^{-1}$, quite good for such an active star.

\section{HD $216489=$ HR $8703=$ IM Peg}

\subsection{Brief history}

Harper $(1920,1935)$ found HR 8703 to be a single-lined spectroscopic binary with an orbital period of 24.649 days. From 1990 to 1992 Oláh et al. (1998) obtained 19 new radial velocities at NSO and KPNO and computed a new orbit with an improved period of 24.64876 days. Herbst (1973) found HR 8703 to have strong CaII H and K emission lines and discovered the light variability of the star. From three seasons of photometry Eaton et al. (1983) found a mean photometric period of 24.39 days. Recently, Strassmeier et al. (1997) obtained a long-term average of 24.494 days from 18 years of $V$-band photometry.

\subsection{Orbital elements}

The Vienna group collected 44 velocities at NSO during a single 1996-97 observing run. Independent orbital solutions for those NSO velocities and for Harper's (1920, 1935) 20 DAO velocities were computed with the SB1 program. From a comparison of those solutions Harper's velocities were given weights of 0.2 , and $-3.0 \mathrm{~km} \mathrm{~s}^{-1}$ was added to each velocity. The velocities of Oláh et al. (1998) from NSO and KPNO, corrected to the Scarfe et al. (1990) velocity system, are poorly distributed in orbital phase. Thus, those velocities were assigned zero weight and included in a combined solution of Harper's velocities and

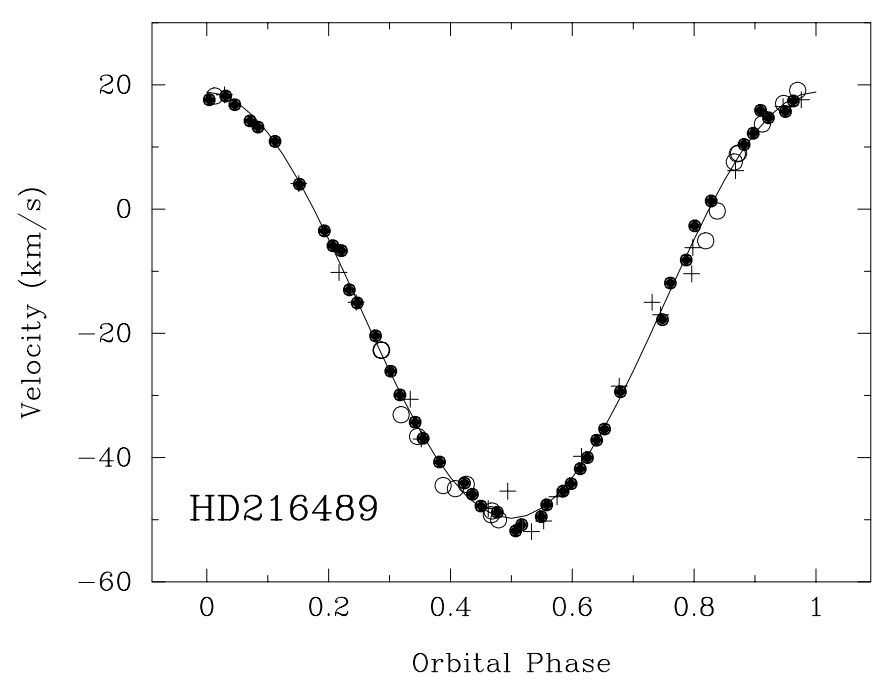

Fig. 12. Radial-velocity curve of HR $8703=$ IM Peg. Filled circles are from NSO, open circles from Oláh et al. (1998), and pluses from Harper $(1920,1935)$

the Vienna group's NSO velocities. As a result, we have given the Oláh et al. (1998) NSO velocities unit weight and added $-3.0 \mathrm{~km} \mathrm{~s}^{-1}$ to those velocities. The three KPNO velocities of Oláh et al. (1998) were also given unit weight, but no velocity correction was applied.

We have assumed that the velocity offsets determined for some of the above data sets result from asymmetric line profiles, as is the case in the most active systems. However, as we have shown for LN Peg and HD 106225, systematic velocity residuals are sometimes due to the presence of a third star. Although 83 velocities of HR 8703 have been obtained, they are not well suited to search for evidence of a long-period orbit since the velocities are extensively clumped around only four epochs. Additional velocities obtained over several years will be needed to confirm our assumption.

An all-data solution with six velocities given zero weight because of their large residuals was computed with SB1. The eccentricity of that solution was quite small, $0.013 \pm 0.008$, and so a circular-orbit solution with SB1C was obtained for comparison. According to the precepts of Lucy \& Sweeney (1971), the orbit is at the borderline of the $5 \%$ level of significance. We have adopted the circularorbit solution, whose elements appear in Table 5. Since the eccentricity is zero, the time of periastron passage has been replaced by the time of maximum velocity. Our radial velocities and velocity residuals to the computed orbit are given in Table A15 of the Appendix. Also listed are the velocities of Harper (1920, 1935) and Oláh et al. (1998). Figure 12 compares the computed orbit with the observed velocities, where zero phase is the time of maximum positive velocity. The standard error of an observation of unit weight is $0.9 \mathrm{~km} \mathrm{~s}^{-1}$, typical of our results for a very active star. 
Acknowledgements. We are grateful to the Austrian Science Foundation (FWF) for support through grant FWF-S7302AST. Special thanks go to Trudy Tilleman at NSO for taking some of the NSO spectra. We thank David Barlow, who generously provided us with copies of his circular-orbit programs. This research has been supported in part by NASA grants NCC5-228 as well as NSF grant HRD-9706268 to Tennessee State University.

\section{References}

Balona L.A., 1987, SAAO Circ. 11, 1

Barker E.S., Evans D.S., Laing J.D., 1967, R. Obs. Bull. 130

Barlow D., 1998 (private communication)

Bergoffen M.J., Stoke J., Walter F., Fleming T.A., 1988, PASP 100, 736

Berdyugina S.V., Jankov S., Ilyin I., Tuominen I., Fekel F.C., 1998, A\&A 334, 863

Bidelman W.P., 1954, ApJS 1, 175

Bidelman W.P., 1981, AJ 86, 553

Bidelman W.P., 1983, AJ 88, 1182

Bidelman W.P., 1991 (private communication)

Bidelman W.P., MacConnell D.J., 1973, AJ 78, 687

Bopp B.W., Stencel R.E., 1981, ApJ 247, L131

Bopp B.W., Goodrich B.D., Africano J.L., Noah P.V., Meredith R.J., Palmer L.H., Quigley R.J., 1984, ApJ 285, 202

Boyd L.J., Genet R.M., Hall D.S., 1984, IBVS, No. 2546

Campbell W.W., 1911, Lick Obs. Bull. 6, No. 199, 140

Cannon J.B., 1915, Publ. Dominion Obs. Ottawa II, No. 6, 141

Collier Cameron A., 1987, SAAO Circ. 11, 13

Cousins A.W.J., Stoy R.H., 1963, R. Obs. Bull. 64

Dadonas V., 1994 (private communication)

Dadonas V., Sperauskas J., Fekel F.C., Morton M.D., 1994, Ap\&SS 220, 97

Daniels W., 1966, University of Maryland, Dept. of Physics and Astronomy Technical Report, No. 579

Donati J.-F., Semel M., Carter B.D., Rees D.E., Cameron A.C., 1997, MNRAS 291, 658

Eaton J.A., Hall D.S., Henry G.W., Lovell L.P., Stephan C.A., Chambliss C.R., Detterline P.K., Landis H.J., Louth H., Renner T.R., Skillman D.R., 1983, Ap\&SS 89, 53

Eggen O.J., 1973, PASP 85, 42

ESA 1997, The Hipparcos and Tycho Catalogues, ESA SP-1200

Evans D.S., Bopp B.W., 1974, Observatory 94, 80

Fekel F.C., 1997, PASP 109, 514

Fekel F.C., Hall D.S., Henry G.W., 1984, IBVS 2543

Fekel F.C., Kirkpatrick J.D., Yang X., Strassmeier K.G., 1989, AJ 97, 202

Fekel F.C., Moffett T.J., Henry G.W., 1986, ApJS 60, 551

Fitzpatrick M.J., 1993, in Hanish R.J., Brissenden R.V.J. \& Barnes J. (eds.), Astronomical Data Analysis Software and Systems II, PASPC 52, p. 472

Fleming T.A., Gioia I.M., Maccacaro T., 1989, AJ 98, 692

Flower P.J., 1996, ApJ 469, 355
Gratton L., 1950, ApJ 111, 31

Grewing M, Bianchi L., Garrido R., 1989, A\&A 223, 172

Harper W.E., 1920, Publ. Dominion Astrophys. Obs. 1, 203

Harper W.E., 1935, Publ. Dominion Astrophys. Obs. 6, 207

Heard J.F., 1956, Publ. of David Dunlap Obs. 2, 107

Hendry E.M., 1980, PASP 92, 825

Henry G.W., Fekel F.C., Hall D.S., 1995, AJ 110, 2926

Herbst W., 1973, A\&A 26, 137

Hoffmann M., 1980, IBVS, No. 1878

Hooten J.T., Hall D.S., 1990, ApJS 74, 225

Houk N., 1982, Michigan Catalogue of 2-Dimensional Spectral Types for the HD Stars. Ann Arbor, U. of Michigan

Jones D.H.P., Fisher J.L., 1984, A\&AS 56, 449

Joy A.H., Wilson R.E., 1949, ApJ 109, 231

Kaye A.B., Hall D.S., Henry G.W., et al., 1995, AJ 109, 2177

Latham D.W., Mazeh T., Carney B.W., McCrosky R.E., Stefanik R., Davis R.J., 1988, AJ 96, 567

Lucy L.B., Sweeney M.A., 1971, AJ 76, 544

Mazeh T., Shaham J., 1979, A\&A 77, 145

O'Neal D., Saar S.H., Neff J.E., 1996, ApJ 463, 766

Oláh K., Marik D., Houdebine E.R., Dempsey R.C., Budding E., 1998, A\&A 330, 559

Parenago P., 1931, Variable Stars (Russian) 3, 99

Pasquini L., Lindgren H., 1994, A\&A 283, 179

Raveendran A.V., Mekkaden M.V., 1998, IBVS, No. 4646

Raveendran A.V., Mekkaden M.V., Mohin S., 1982, MNRAS 199, 707

Rodonó M., Cutispoto G., Messina S., 1994, A\&A 281, 756

Scarfe C.D., Batten A.H., Fletcher J.M., 1990, Publ. Dominion Astrohys. Obs. 18, 21

Schmidt-Kaler Th., 1982, in Landolt-Börnstein, Vol. I/2b, p. 15

Söderhjelm S., 1984, A\&A 141, 232

Spencer Jones H., 1928, Annals Cape Obs. 10, part 8, 1

Stebbins J., 1928, Publ. Washburn Obs. 15, 29

Stockton R.A., Fekel F.C., 1992, MNRAS 256, 575

Strassmeier K.G., 1994a, A\&AS 103, 413

Strassmeier K.G., 1994b, A\&A 281, 395

Strassmeier K.G., 1996, A\&A 314, 558

Strassmeier K.G., Bartus J., Cutispoto G., Rodonó M., 1997, A\&AS 125, 11

Strassmeier K.G., Fekel F.C., Bopp B.W., Dempsey R.C., Henry G.W., 1990, ApJS 72, 191

Strassmeier K.G., Hall D.S., Boyd L.J., Genet R.M., 1989, ApJS 69, 141

Strassmeier K.G., Hall D.S., Fekel F.C., Scheck M., 1993, A\&AS 100, 173

Strassmeier K.G., Rice J.B., 1998, A\&A 330, 685

Udry S., 1998 (private communication)

Vogt S.S., Penrod G.D., 1983, PASP 95, 565

Weber M., Strassmeier K.G., 1998, A\&A 330, 1029

Wiesniewski W.Z., 1973, MNRAS 161, 331

Wolfe R.H., Horak H.G., Storer N.W., 1967, in: M. Hack (ed.), Modern Astrophysics, Gordon \& Breach, New York, p. 251

Woolley R., Penston M.J., Harding G.A., Martin W.L., Sinclair J.E., Haslam C.M., Aslan S., Savage A., Aly K., Asaad A.S., 1981, R. Obs. Ann. 14

Zappa F., 1974, CD: Live in New York 